\title{
National Drug Formulary review of statin therapeutic group using the multiattribute scoring tool
}

This article was published in the following Dove Press journal:

Therapeutics and Clinical Risk Management

3 December 2013

Number of times this article has been viewed

\author{
Azuana Ramli ${ }^{1,3}$ \\ Syed Mohamed Aljunid ${ }^{1,2}$ \\ Saperi Sulong ${ }^{2}$ \\ Faridah Aryani Md Yusof ${ }^{3}$ \\ 'United Nations University \\ International Institute for Global \\ Health (UNU-IIGH), Kuala Lumpur, \\ Malaysia; ${ }^{2}$ International Centre for \\ Casemix and Clinical Coding (ITCC), \\ Universiti Kebangsaan Malaysia \\ Medical Centre, Kuala Lumpur, \\ Malaysia; ${ }^{3}$ Pharmaceutical Services \\ Division, Ministry of Health, Petaling \\ Jaya, Malaysia
}

Purpose: HMG-CoA reductase inhibitors (statins) are extensively used in treating hypercholesterolemia. The statins available in Malaysia include atorvastatin, lovastatin, pravastatin, rosuvastatin, simvastatin, and fluvastatin. Over the years, they have accumulated in the National Drug Formulary; hence, the need for review. Effective selection of the best drugs to remain in the formulary can become complex due to the multiple drug attributes involved, and is made worse by the limited time and resources available. The multiattribute scoring tool (MAST) systematizes the evaluation of the drug attributes to facilitate the drug selection process. In this study, a MAST framework was developed to rank the statins based on their utilities or benefits.

Methods: Published literature on multicriteria decision analysis (MCDA) were studied and five sessions of expert group discussions were conducted to build the MAST framework and to review the evidence. The attributes identified and selected for analysis were efficacy (clinical efficacy, clinical endpoints), safety (drug interactions, serious side effects and documentation), drug applicability (drug strength/formulation, indications, dose frequency, side effects, food-drug interactions, and dose adjustments), and cost. The average weights assigned by the members for efficacy, safety, drug applicability and cost were $32.6 \%, 26.2 \%, 24.1 \%$, and $17.1 \%$, respectively. The utility values of the attributes were scored based on the published evidence or/and agreements during the group discussions. The attribute scores were added up to provide the total utility score.

Results: Using the MAST, the six statins under review were successfully scored and ranked. Atorvastatin scored the highest total utility score (TUS) of 84.48 , followed by simvastatin (83.11). Atorvastatin and simvastatin scored consistently high, even before drug costs were included. The low scores on the side effects for atorvastatin were compensated for by the higher scores on the clinical endpoints resulting in a higher TUS for atorvastatin. Fluvastatin recorded the lowest TUS.

Conclusion: The multiattribute scoring tool was successfully applied to organize decision variables in reviewing statins for the formulary. Based on the TUS, atorvastatin is recommended to remain in the formulary and be considered as first-line in the treatment of hypercholesterolemia.

Keywords: multicriteria decision analysis, utility score, drug attributes, drug selection

\section{Introduction}

The need for new drugs continues to rise, often without a corresponding increase in the drug budgets allocated to hospitals. The budget provided needs to be wisely distributed to accommodate the needs and to maximize the benefits that can be obtained. A drug formulary system is fundamental to managing the utilization of medicines and expenditure in any health institution. A carefully developed and maintained drug formulary 
promotes the rational use of medicines by allowing patients access to only cost-effective and safe medicines. ${ }^{1}$ In addition to managing the expenditures, this should ultimately improve the quality of health care received by the patients. However, without stringent control, drugs in the formulary tend to accumulate over time. This happens when superior drugs that enter the formulary are not followed by a removal of the inferior ones.

In Malaysia, the Ministry of Health (MOH) Drug Formulary (Formulari Ubat Kementerian Kesihatan Malaysia [FUKKM]) or the "Blue Book" lists the drugs approved for use in all the $\mathrm{MOH}$ institutions. Adding or deleting drugs to or from the FUKKM are made through a national committee, the National Drug List Review (NDLR) panel. The NDLR panel members include consultants and specialists from various disciplines, pharmacists, and health administrators. ${ }^{2}$ Drug listing applications are reviewed and the evaluation reports are prepared by drug evaluators. These reports are later sent to the NDLR panel members. Besides these reports, the NDLR panel also receives inputs from a technical working committee, which includes specialists and experts concerned with the related disease, to guide their discussions in making decisions for listing during the NDLR panel meetings.

Currently, the drug evaluators are staff members of the Formulary and Pharmacoeconomic Unit of the Pharmaceutical Services Division (PSD), who are not members of the NDLR panel. They are generally trained in critical appraisals, pharmacoeconomics, and evidence-based medicines (personal communications). Evaluating drugs effectively can be an arduous task. Besides, they need to produce the expected functions within the limits of minimum access to the literature and time available.

On the other hand, it may not be easy for the NDLR panel members to make decisions to select drugs for the formulary due to the sheer volume of information available for each drug being considered. Drug review reports prepared by the drug evaluators often become copious documents that can take time to read. This is further aggravated by the complexity of the process of drug selection. Different drugs have different sets of values for the different criteria being considered. Additionally, decisions can be influenced by 'emotional/irrational' factors including panel members' personal experience with the drug use, relationship with pharmaceutical companies, and even a few "unconscious" criteria. ${ }^{3}$ The unconscious criteria affecting decision makers happens mainly as a result of heavy advertising campaigns by pharmaceutical companies. Unconsciously, certain drug names felt more superior due to their greater presence on advertising materials like note pads, pens, calendars etc. As a result, drug selection for the formulary is often associated with lack of transparency, poor rationalization, and bias.

Multicriteria decision analysis (MCDA) provides a structured approach in complex decision making situations when alternative options have multiple criteria with differing values to consider. This approach has been used regularly to make decisions and choices in the fields of social science, engineering, and environment. ${ }^{4}$ With MCDA, the criteria are weighted according to their importance, the alternatives available are then evaluated against these criteria. ${ }^{5}$ The weighted score for each criterion is obtained by multiplying the weights with the value scores of the criterion. At the end of the process, the total scores of each alternative are obtained by aggregating the weighted scores of all the criteria evaluated. These overall coherent values of the alternatives are made available to the decision makers to assist them in making better evidence-based decisions. ${ }^{6}$

Evaluating and selecting drugs for the formulary necessitates applying the MCDA as multiple attributes including efficacy, safety and cost with different degrees of importance needing to be considered. ${ }^{7}$ MCDA has been successfully used in medical fields elsewhere to evaluate treatment options and drug selection. ${ }^{8-11}$ In The Netherlands, Janknegt et al developed a scoring system called the System of Objectified Judgement Analysis (SOJA), for drug selection. This system has been a proven help to guide decision makers in arriving at more rational decisions. ${ }^{12}$ It has also been shown to provide cost savings to health care providers in the UK. ${ }^{13}$ Similar tools have also been used in Thailand to facilitate evidencebased drug selection processes for the National Essential Drug Lists with implementation of the information, Safety, administration restrictions, frequency of administrations and Efficacy (iSafE) scoring system. ${ }^{14}$

In the case of FUKKM, the number of drugs within many therapeutic groups has accumulated over the years. Directly adopting the scoring tools used elsewhere, however, was not practical. Primarily, detailed descriptions of the tools available in published literature were insufficient to replicate them. To directly adopt some of the more established tools, fees were required to be paid and adjustments were also needed for different settings. iSafE was set in Thai language and drug utility measurements were found to be more complex. ${ }^{14}$ From our assessments, the most suitable features of a tool for our settings may not be found in one particular tool but distributed among the various tools. Thus, a modified tool will fulfill PSD's needs in providing simple and concise evidence based reviews to decision makers. Additionally, we 
foresee the advantages of fostering ownership and interests of the tool, as well as providing an in-depth grasp of the tool framework in the tool development process. By applying the MCDA principles, the scoring tool will enable drug evaluators to summarize their evidence-based evaluations on drug alternatives, systematically and simply. The coherent scores, in turn, could guide the members of the NDLR panel to compare and rank the alternatives systematically, during their discussions. Thus, more rational and transparent decisions can be achieved in streamlining the formulary list.

In this work, we report on the development of a local scoring tool framework based on the MCDA principles, and study its application by reviewing a drug group in the national formulary. The statin drug group was selected by the working committee to pilot this scoring framework. Statin or 3-hydroxy -3-methylglutaryl-coenzyme A (HMG-CoA) reductase inhibitor is the standard treatment for hypercholesterolemia, a major risk factor for cardiovascular diseases. Based on the earlier national utilization study, statin consumption in the country is high, due to the high prevalence of hypercholesterolemia. Based on the Malaysian Statistics on Medicines (2008), lipid modifying agents, including mainly statins, were the fifth most used drugs in Malaysia in 2008, surpassed only by anti-diabetics, renin-angiotensin blockers, calcium channelblockers and beta-blockers. ${ }^{15}$ The statins registered for use in Malaysia include atorvastatin, lovastatin, pravastatin, rosuvastatin, simvastatin, and fluvastatin. Over the years, all these statins (except fluvastatin) have been added to the FUKKM. Three of these statins namely, lovastatin, simvastatin, and atorvastatin have also occupied the top 40 most-used drugs in $2008 .^{15}$ It was decided that a review of the statin list in the FUKKM is judicious to guide prescribers in choosing the most cost-effective statin. The review is deemed necessary as most of the statins are now available in the generic forms and the costs of these statins have changed from the time they were first listed in the formulary. In this study, the six statins available were evaluated, scored, and ranked using the locally developed MAST framework.

\section{Methods}

A working committee of eleven members under the leadership of researcher AR was formed. The main responsibilities of the committee were to design and produce a suitable local scoring framework for drug selection. The tool is targeted for use by drug evaluators in providing information to the local decision makers (members of the NDLR panel). Therefore, the expert committee mainly comprised of drug evaluators and pharmacists who are familiar with the data requirements of the NDLR panel. It includes a pharmacist trained in pharmacoeconomics (FAMY), three drug reviewers (Haarathi Chandriah, also trained in clinical pharmacy, Rosliza Lajis, and Hariana Haris), a hospital pharmacist (Lim Ming Tsuey), three pharmacists working at the National Formulary Unit (Azuwana Supian, Noraini Saari, and Hayati Alwani), a pharmacy administrator (Anis Talib) who is also the secretary to the NDLR panel, and a family medicines specialist (Dr Aznida Abd Aziz) for relevant clinical consultations. The group met five times between January and July 2012 to build the present multiattribute scoring tool (MAST) based on the MCDA principles. Published literature on this theory and studies available that have applied similar methods in drug selection were reviewed and discussed. The requirements and concerns of the drug evaluators who would be applying the tool when evaluating drugs were also considered. The tool needed to be able to function within the limited resources available to the drug evaluators in their workplace as well as be simple to understand and use. To score the drug utilities, the committee decided to primarily begin with the evidence provided by the drug information database, Micromedex (Thomson Reuters, Greenwood Village, CO, USA). Pharmaceutical Services Division has subscribed to Micromedex for many years. It is mainly used by drug evaluators and drug information pharmacists for quick and comprehensive, reliable, and updated drug information. Micromedex was ranked as one of the best performing drug databases in terms of scope, completeness, and easy usability in an analysis of online drug information databases. ${ }^{16}$ Additional references were searched for and discussed when the information available in Micromedex was insufficient or inconclusive.

The finalized steps agreed upon by the committee to be used in evaluating and scoring the drugs for the formulary have been discussed below. These steps have been developed mainly based on the MCDA steps and other reported works of its applications. ${ }^{6,8-10,12,17}$ The review on the drugs was performed from the viewpoint of the Health Service Provider, Pharmaceutical Services Division, Ministry of Health. Ethical approval to conduct the study was obtained from the Medical Research Ethics Committee, National Institutes of Health, MOH, Malaysia.

\section{Step I: select drug class and list the drugs to be reviewed}

In this first step, the therapeutic class to be reviewed using the scoring tool and all drugs eligible to be scored within that group were selected. The Anatomical Therapeutic Classification (ATC) and the drug indications were also referred to confirm the drugs selected. ${ }^{18}$ 


\section{Results}

In its first meeting, the working group agreed to review the HMG Co-A reductase inhibitor (statins) therapeutic class to pilot the scoring tool framework being developed. All the drugs in this drug class (ATC Code C10AA) registered with the Malaysian National Pharmaceutical Control Bureau (NPCB) were selected for review. The six statins chosen were atorvastatin, simvastatin, pravastatin, lovastatin, rosuvastatin (listed in FUKKM) and fluvastatin (registered in Malaysia, but not in the FUKKM list). The strengths and formulation locally available were searched using the Quest 2 search engine on the NPCB website at http://portal.bpfk.gov.my/. The list of drugs and the information collected were tabled in a Microsoft Excel worksheet.

\section{Step 2: determine the selection criteria}

In this second step, the common drug attributes and selection factors (criteria) against which the drugs were to be quantified and scored were determined, based on the published literature of similar work , $^{6,9,14,17}$ and group discussions. The committee discussed all the possible selection factors and decided on the relevant factors for drug selection of the statin therapeutic group. Further discussion was conducted to ensure that the list of factors was complete and there was no duplication. A worksheet for each factor to be evaluated and scored was then prepared in Microsoft Excel.

\section{Results}

The main selection attributes agreed upon by the committee to be significant in selecting drugs in the statin groups include efficacy, safety, drugs' applicability (include patients' convenience) and economics (Table 1). A total of 14 worksheets were prepared; one for factor weights, twelve for the 12 criteria, and one for total scores.

Two factors (sub-criteria) for efficacy decided upon by the committee to be considered for the evaluation were clinical efficacy/effectiveness measured in percentage reduction in low-density lipoprotein cholesterol (LDL-C) and medium/long-term effect (clinical endpoints). LDL-C measurement was selected as it is the most common intermediate measure for the efficacy of the statins in randomized controlled trials. The LDL-C levels have also been proven to be directly related to risks of coronary heart disease ${ }^{19}$ For clinical endpoints, the utilities of the statins in primary and secondary prevention of cardiovascular events were also evaluated. ${ }^{20}$

Selection factors considered under the safety attribute were serious side effects (for example myopathy, rhabdomyolysis, Stevens-Johnson Syndrome, dermatomyositis and liver failure), drug-drug interactions and available documentations on the drugs reviewed. For drug applicability (and patient convenience) the selection factors considered were the drug approved indications, available formulations, dose frequencies, interactions with food, dose adjustments in special populations (pregnant women, elderly and children, and those having renal deficiency and liver problems) and minor but frequent side effects. Based on the physician's input, side effects considered minor include headache, dizziness, indigestion, cough, and rash.

In the economic criterion, as local pharmacoeconomic data are unavailable, drug costs and additional costs (for example administration costs, lab costs) that can be incurred

Table I Weights assigned to the selection criteria by members of the working committee

\begin{tabular}{|c|c|c|c|c|c|c|c|c|c|}
\hline Attributes & Selection criteria (factors) & AR & $\mathbf{H H}$ & NS & FAMY & $\mathbf{R L}$ & $\mathrm{HC}$ & LMT & Average weight \\
\hline \multirow[t]{3}{*}{ Efficacy } & Clinical efficacy & 16 & 10 & 20 & 22 & 13 & 13 & 17 & 15.9 \\
\hline & Medium/long term effects & 19 & 10 & 20 & 15 & 17 & 18 & 18 & 16.7 \\
\hline & & \multicolumn{7}{|c|}{ Weight subtotal (efficacy) } & 32.6 \\
\hline \multirow[t]{4}{*}{ Safety } & Drug interactions & 12 & 5 & 10 & 5 & 10 & 8 & 10 & 8.6 \\
\hline & Serious side effects & 10 & 10 & 10 & 10 & 10 & 10 & 10 & 10.0 \\
\hline & Documentations & 8 & 5 & 10 & 10 & 5 & 10 & 5 & 7.6 \\
\hline & & \multicolumn{7}{|c|}{ Weight subtotal (safety) } & 26.2 \\
\hline \multirow[t]{7}{*}{ Drug applicability } & Formulations and drug strength & 0 & 10 & 2 & 5 & 5 & 6 & 2 & 4.3 \\
\hline & No of approved indications & 7 & 5 & 5 & 5 & 5 & 5 & 7 & 5.6 \\
\hline & Dose frequency & 2 & 0 & 2 & 0 & 3 & 3 & 2 & 1.7 \\
\hline & Frequent (but not serious) side effects & 6 & 5 & 3 & 8 & 4 & 4 & 5 & 5.0 \\
\hline & Interaction with food & 5 & 5 & 1 & 5 & 2 & 3 & 2 & 3.3 \\
\hline & Dose adjustments & 5 & 5 & 2 & 5 & 6 & 5 & 2 & 4.3 \\
\hline & & \multicolumn{7}{|c|}{ Weight subtotal (patient acceptability) } & 24.2 \\
\hline \multirow[t]{2}{*}{ Economics } & Costs & 10 & 30 & 15 & 10 & 20 & 15 & 20 & I7.I \\
\hline & Total & 100 & 100 & 100 & 100 & 100 & 100 & 100 & 100 \\
\hline
\end{tabular}

Note: Figures in bold are weight subtotal for each attribute, for example 32.6 is the weight subtotal for efficacy.

Abbreviations: AR, Azuana Ramli; HH, Hariana Haris; NS, Noraini Saari; FAMY, Faridah Aryani Md Yusof; RL, Rosliza Lajis; HC, Haarathi Chandriah; LMT, Lim Ming Tsuey. 
when using the drugs were considered. The committee noted that, if available, the results from the local pharmacoeconomic studies should be considered when scoring the economic attribute. However, care must be taken so as not to overlap the costs because pharmacoeconomic studies normally include the costs mentioned above.

\section{Step 3: assign weights to selection criteria}

Once the selection criteria against which to evaluate the drugs had been agreed upon by the committee each criterion was assigned a weight. Each member in the working group (seven of the eleven members attended this meeting) was asked to assign a weight score to each criterion individually listed in a table in a pre-prepared form. Higher scores were assigned to the more important criteria. Total weight scores needed to total to 100 . The members completed the weightings in a twostep process. First, a total weight score of 100 was assigned to the four main attributes (efficacy, safety, drug applicability and economics). Then, the weight scores for each selection criterion under the main attributes were assigned accordingly. For example if efficacy was assigned a weight of 35 (out of a possible 100), the two criteria under efficacy, which were 'clinical efficacy' and 'medium/long-term effects' could be given a weightage of 15 and 20 , respectively, which totaled to 35 . The weights assigned by each member were then tabled and openly discussed in the group meeting to clear any misunderstandings. Each individual was allowed to amend their scores, if they deemed fit, following further group deliberations. Detailed instructions on weight assignment are recorded in the Supplementary materials.

\section{Results}

During the second meeting, all the weights assigned by the individual members were collected and averaged for each criterion to be used as the final weightages (base case) for this scoring exercise. The list of criteria used to evaluate the drugs and the average weights assigned, agreed upon by the group members, are shown in Table 1.

\section{Step 4: decide on comparative measures to evaluate each selection criterion and plan a common utility scale for the criteria}

For each selection criterion being evaluated, the comparative measures (measures of drug values/utility) needed to be determined. For example, for the selection criterion 'efficacy' for a particular statin, the committee was required to decide whether to use \% reduction in the LDL-C or total cholesterol or the percentage of patients who achieved the target LDL (or total cholesterol level) based on the availability of these data (for all the drugs evaluated) from clinical trials or review papers selected.

Then, the utility scale to be used for each selection criterion was constructed. This common utility scale across all criteria was to ensure that the criteria weightages could function as intended and the scores could be appropriately added up to arrive at the final score for each drug, later on in the scoring process. Thus, the total scores could be compared among the alternative drugs. With reference to the previous work, ${ }^{6,8}$ the committee decided that the utility scale should range from 0 (lowest score) to 100 (highest score). Simple mathematical formulae to determine the utility values/scores of each drug on this scale (0-100) were adopted from the previous work on application of the MCDA.., 8

For example, to compare the clinical efficacy of the statin drugs, a literature search was conducted to determine the range of the possible values of percentage reduction in the LDL across all the statin drugs being considered. The worst value in the range, $\mathrm{V}_{\min }$, needed to correspond to score 0 on the utility scale while the highest, $\mathrm{V}_{\max }$, corresponded to 100. In this case, however, to accommodate more drugs that could be evaluated at a later date which could have a worse value than $\mathrm{V}_{\text {min }}$, the working group decided that the score 0 for the utility scale be placed at $80 \%$ of $\mathrm{V}_{\text {min }}(20 \%$ lower than the actual $\mathrm{V}_{\min }$ ). The same was done at the high end of the utility scale, where $20 \%$ more was added to the $\mathrm{V}_{\text {max }}$ corresponding to score 100 on the utility scale to accommodate the new drugs later on that may have a higher efficacy value (very likely). The mathematical representations are presented as Formula (1) and Formula (2), to follow.

\section{Step 5: assign/calculate utility score of each selection criterion for every drug under evaluation}

To calculate the utility score of a drug being considered for this criterion $(\mathrm{U} f)$ on the utility scale developed, the formula given below is used, where $f$ is the actual reading or the raw measurement (in this example it is \% reduction in LDL), reported in the literature/s selected regarding the particular drug:

$$
U f=\frac{100\left(f-\left[V_{\min }-20 \%\right]\right)}{\left(\left[V_{\max }+20 \%\right]-\left[V_{\min }-20 \%\right]\right)}
$$


The formula given above was used for criteria that have a roughly linear relationship between the criteria values and the utility scores, namely efficacy and drug applicability. For factor measurements with inverse relationships to the utility scores including cost, side effects and drug interactions, the formula given below was used instead:

$$
U f=\frac{100\left(\left[V_{\max }+20 \%\right]-f\right)}{\left(\left[V_{\max }+20 \%\right]-\left[V_{\min }-20 \%\right]\right)}
$$

The strengths of the evidence regarding efficacy and effectiveness provided by Micromedex (MM) were factored in when scoring these criteria.

For criteria without any reported values from trials or studies, the committee members brainstormed, discussed and decided by consensus on the appropriate allocation of utility or location scores from 0 to 100 , based on other sources, any previous work or reviews on the subjects and knowledge of current practices. Applying the formulae accordingly, the scores are calculated on Microsoft Excel worksheets using one sheet for each criterion evaluated.

\section{Results (step 4 and step 5) Efficacy Clinical efficacy}

The committee unanimously decided to use the mean percentage reduction in the LDL-C values to score clinical efficacies. After checking through landmark trials reported by TrialResults Centre website at http://www.trialresultscenter.org/,, ${ }^{21}$ drug evaluations and comparisons of the statins in $\mathrm{MM},{ }^{20}$ as well as other meta-analysis and review papers available on statins and discussing them, the committee decided to use the efficacy values reported in STELLAR (Statin Therapies for Elevated Lipid Levels compared Across doses to Rosuvastatin) trial $^{22-24}$ to score these criteria. STELLAR was reported to be the most comprehensive trial on the effectiveness of the widely used statins to date. ${ }^{22}$ This was a 6-week open-label, parallel group, multicenter trial involving 2,431 adults with hypercholesterolemia randomized to rosuvastatin 10,20 , and $40 \mathrm{mg}$, atorvastatin $10,20,40$, and $80 \mathrm{mg}$, pravastatin 10,20 , and $40 \mathrm{mg}$ or simvastatin $10,20,40$, and $80 \mathrm{mg}$. The primary end point (change in plasma LDL-C concentration from baseline to six weeks) was used to compare the statin efficacies. Based on the defined daily doses (DDD) of the individual statins, the group concluded on appropriate raw data values (percentage reduction in LDL-C) to be used for scoring. The values ranged from $27 \%$ (for pravastatin, DDD $30 \mathrm{mg}$ ) and $46 \%$ (for rosuvastatin, DDD $10 \mathrm{mg}$ ). To score lovastatin and fluvastatin, which were omitted from the STELLAR trial, the committee made reference to a meta-analysis on the efficacy of the statins conducted by Edwards and Moore. ${ }^{25}$ Based on this analysis, the percentage reduction LDL-C of $31 \%$ and $27 \%$ were used for lovastatin and fluvastatin, respectively. The committee agreed that this was acceptable as the percentages of the LDL-C reduction reported in this meta-analysis of the other 4 statins (atorvastatin, simvastatin, pravastatin, and rosuvastatin) were similar to the findings of the STELLAR trial. Further review of the additional data reported in MM based on a cross-sectional survey of 17 general practices in Trent, UK, on a proportion of patients achieving serum concentrations of lower or equal to $5 \mathrm{mmol} / \mathrm{L}$, confirmed concurrence with the results from the STELLAR trial. ${ }^{23,26}$

Substituting these figures into the formula given above, the efficacy scores for the individual drugs under evaluation were determined. As the evidence was obtained from the same trial, the strength of evidence score was kept at 1 . Thus, there were no changes in the scores even when the strengths of the evidence were factored in.

The summary of the results and the percentage of the LDL readings to be considered in the scorings agreed upon by the group are summarized in Table 2 . For example, the calculated utility score using the formula given above for

Table 2 Table of the clinical efficacy scores of statins

\begin{tabular}{|c|c|c|c|c|c|c|c|}
\hline Drug & $\begin{array}{l}\text { Drug dose in } \\
\text { chosen trial } \\
\text { (mg/day) }\end{array}$ & $\begin{array}{l}\text { Mean reduction } \\
\text { in LDL-C in } \\
\text { trials (\%) }\end{array}$ & $\begin{array}{l}\text { Defined } \\
\text { daily dose }\end{array}$ & $\begin{array}{l}\% \text { LDL-C } \\
\text { reduction (to } \\
\text { input in scoring) }\end{array}$ & $\begin{array}{l}\text { Strength } \\
\text { of evidence } \\
\text { score }\end{array}$ & $\begin{array}{l}\text { Calculated } \\
\text { utility score } \\
\text { (efficacy) }\end{array}$ & $\begin{array}{l}\% \text { of patients } \\
\text { obtained } \\
\text { LDL-C goal }{ }^{22,23, *}\end{array}$ \\
\hline Pravastatin ${ }^{23}$ & $10-40 \mathrm{mg}$ & $20-29$ & $30 \mathrm{mg}$ & 27 & 1 & 58.0 & $31-55$ \\
\hline Simvastatin $^{23}$ & $10-40 \mathrm{mg}$ & $28-39$ & $30 \mathrm{mg}$ & 37 & 1 & 72.9 & $51-66$ \\
\hline Lovastatin ${ }^{25}$ & $40 \mathrm{mg}$ & 30 & $45 \mathrm{mg}$ & 31 & 1 & 64.0 & NA \\
\hline Atorvastatin ${ }^{23}$ & $10-80 \mathrm{mg}$ & $37-51$ & $20 \mathrm{mg}$ & 42 & 1 & 80.4 & $69-82$ \\
\hline Rosuvastatin ${ }^{23}$ & $10-40 \mathrm{mg}$ & $46-55$ & $10 \mathrm{mg}$ & 46 & 1 & 86.3 & $82-89$ \\
\hline Fluvastatin ${ }^{25}$ & $40 \mathrm{mg}$ & 24 & $60 \mathrm{mg}$ & 27 & 1 & 58.0 & NA \\
\hline
\end{tabular}

Note: $*$ This information is tabled to confirm the relative efficacies of the statins compared.

Abbreviations: LDL-C, low-density lipoprotein cholesterol; NA, not available. 
pravastatin efficacy is shown below. Assuming reasonably small differences among the efficacies of the drugs in this therapeutic group, the committee decided to score the drugs using the $50 \%$ upper end (fiftieth percentile) of the location score range.

$$
\begin{aligned}
U f & =50+\frac{50\left(f-\left[V_{\min }-20 \%\right]\right)}{\left(\left[V_{\max }+20 \%\right]-\left[V_{\min }-20 \%\right]\right)} \\
U f & =50+\frac{50(27-[27 \times 0.8])}{([46 \times 1.2]-[27 \times 0.8])} \\
& =58
\end{aligned}
$$

\section{Medium-/long-term effect (clinical endpoints)}

Data from MM on medium- or long-term effects of statins related to the prevention of cardiovascular morbidity and mortality were studied. Relevant trials were sought in TrialResults Centre via (http://www.trialresultscenter.org/) $)^{21}$ and reviewed. Evaluating these criteria was challenging. The populations studied in the various trials differed greatly making direct comparisons based on the trials among the statins difficult and sometimes quite unsuitable. The relevant information is revealed in Table 3. Additionally, reported measurements for the clinical endpoints were not standardized; these include coronary events, cardiovascular events, all causes of death, coronary death, major adverse cardiac events (which could also differ in definition from one trial to the other) and so on.

Consequently, the committee decided to score this criterion based on the information summarized and conclusions drawn from MM (Table 3). Evidence of statin use for primary and secondary preventions of adverse cardiovascular events were considered both with respect to strength of the recommendation and strength of the evidence. Strengths of recommendations are categorized into Class I (recommended), Class IIA (recommended in most cases), Class IIB (recommended in some cases), and Class III (not recommended). For strength of evidence, evidences based on data derived from meta-analyses of randomized controlled trials with homogeneity with regards to directions and degrees of results between individual studies as well as evidences from multiple randomized control trials involving large number of patients are categorized into Category A. Evidences based on data derived from meta-analyses of randomized controlled

\begin{tabular}{|c|c|c|c|c|c|c|}
\hline Drug & $\begin{array}{l}\text { Trial reference } \\
\text { N: studied/ } \\
\text { control }\end{array}$ & Population studied & $\begin{array}{l}\text { Reported } \\
\text { ARR of } \\
\text { CVE (\%) }\end{array}$ & $\begin{array}{l}\text { \# R/S for } 1^{\circ} \\
\text { prophylaxis } \\
\text { of CVE }\end{array}$ & $\begin{array}{l}\text { \# R/S for } 2^{\circ} \\
\text { prophylaxis } \\
\text { of CVE }\end{array}$ & $\begin{array}{l}\text { Calculated } \\
\text { utility score }\end{array}$ \\
\hline Pravastatin & $\begin{array}{l}\text { MEGA } 2006^{39} \\
\text { N: } 3,866 / 3,966 \\
\text { PROSPER } 2002^{40} \\
\text { N: } 2,891 / 2,913\end{array}$ & $\begin{array}{l}\text { Hypercholesterolemic and no history of CHD } \\
\text { or stroke } \\
\text { Age } 70-82 \text { years; either pre-existing or raised } \\
\text { risk vascular disease: TC of } 4.0-9.0 \mathrm{mmol} / \mathrm{L}\end{array}$ & 2.25 & $\begin{array}{l}\text { Adult IIA/ } \\
\text { category A }\end{array}$ & IIA/B & 79.17 \\
\hline Simvastatin & $\begin{array}{l}\text { HPS } 2002^{41} \\
N: 10,269 / 10,267\end{array}$ & $\begin{array}{l}\text { Age } 40-80 \text { years with CHD, other occlusive } \\
\text { arterial disease or DM }\end{array}$ & 3.06 & $\begin{array}{l}\text { Adult IIA/ } \\
\text { category A }\end{array}$ & $\| \mathrm{A} / \mathrm{A}$ & 84.85 \\
\hline Lovastatin & $\begin{array}{l}\text { AFCAPS/ } \\
\text { TEXCAPS } 1998^{42} \\
\text { N: } 3,304 / 3,301\end{array}$ & $\begin{array}{l}\text { Without clinically evident atherosclerotic CVD } \\
\text { with average TC and LDL-C levels }\end{array}$ & 1.58 & $\begin{array}{l}\text { Adult IIA/ } \\
\text { category A }\end{array}$ & IIA/B & 79.17 \\
\hline Atorvastatin & $\begin{array}{l}\text { CARDS } 2004^{44} \\
\text { N: I,429/I,4I } 2\end{array}$ & $\begin{array}{l}\text { Had had a stroke or TIA within one to six months } \\
\text { before study entry, LDL-C levels of } 2.6 \text { to } \\
4.9 \text { mmol/L, and had no known CHD } \\
\text { With type } 2 \text { DM without elevated LDL-C and } \\
\text { at least one of retinopathy, albuminuria, current } \\
\text { smoking, or hypertension }\end{array}$ & 3.2 & $\begin{array}{l}\text { Adult IIB/ } \\
\text { category B }\end{array}$ & I/A & 84.85 \\
\hline Rosuvastatin & $\begin{array}{l}\text { CORONA } 2007^{45} \\
\text { N: } 2,514 / 2,497 \\
\text { JUPITER } 2008^{46} \\
\text { N: } 8,901 / 8,901\end{array}$ & $\begin{array}{l}\text { At least } 60 \text { years of age with NYHA class II, III, } \\
\text { or IV ischemic, systolic heart failure } \\
\text { Healthy individuals with low LDL-C levels but } \\
\text { elevated C-reactive protein }\end{array}$ & $\begin{array}{l}1.8 \\
0.83\end{array}$ & $\begin{array}{l}\text { Adult IIB/ } \\
\text { category A }\end{array}$ & $\mathrm{IIB} / \mathrm{B}$ & 60.23 \\
\hline Fluvastatin & $\begin{array}{l}\text { HYRIM }{ }^{47} \\
\text { N: } 283 / 285\end{array}$ & $\begin{array}{l}\text { Drug-treated hypertensive men age } 40-74 \text { years } \\
\text { with Total-C } 4.5-8.0 \mathrm{mmol} / \mathrm{L}, \mathrm{BMI} 25-35 \mathrm{~kg} / \mathrm{m}^{2} \text {, } \\
\text { and a sedentary lifestyle }\end{array}$ & 1.38 & $\begin{array}{l}\text { Adult IIB/ } \\
\text { category B }\end{array}$ & IIA/B & 67.80 \\
\hline
\end{tabular}
trials with conflicting conclusions with regards to directions

Table 3 Medium/long-term effect (clinical endpoints) scores

Notes: The trial list is not exhaustive. Results of trials are mainly accessed through Trials-Results Centre website at http://www.trialresultscenter.org/. "Information Source, Micromedex.

Abbreviations: ARR, absolute risk reduction; CVD, cardiovascular disease; CVE, cardiovascular events; R/S, recommendation and strength of evidence; TC, total cholesterol; LDL, low density lipoprotein; CHD, coronary heart disease; TIA, transient ischemic attack; NYHA, New York Heart Association functional classification for heart failure. 
and degrees of results between individual studies as well as randomized control trials that involved small number of patients or had significant methodological flaws (eg, bias, drop-out rate, flawed analysis, etc) and nonrandomized studies (eg, cohort studies, case-control studies, observational studies) are categorized into Category B. ${ }^{20}$ Acknowledging the fact that statins best benefit those with a higher risk of cardiovascular diseases,${ }^{27}$ more weightage was given to the scorings of secondary preventions by $60: 40$.

\section{Safety}

\section{Drug Interactions}

MM was used to obtain information on drug interactions. Eight different types of risks were identified in MM including moderate/probable, moderate/established, major/theoretical, major/probable, major/established, contraindicated/theoretical, contraindicated/probable and contraindicated/established and were assigned risk factors of 0.3 to 1,1 being the highest risk (contraindicated/established). Based on these risks, the drugs were scored according to the number of possible interactions documented in MM and the risk factors involved. Based on this criterion, pravastatin, simvastatin, lovastatin, atorvastatin, rosuvastatin and fluvastatin scored 83.09, 53.45, 59.16, 78.11, 87.71, and 84.79, respectively.

\section{Serious side effects}

The committee explored both Medscape Reference ${ }^{28}$ and $\mathrm{MM}^{20}$ when scoring the side effects but later agreed to refer to the information provided by MM while scoring serious side effects for the drugs evaluated to be consistent with the other criteria. The occurrence of serious side effects of $1 \%$ or more was classified as more common and those less than $1 \%$ as less common and were ranked as risk factors of 1 and 0.3 , respectively. This information was used to mark the raw scores of the serious side effects for these drugs. Using Formula 2, the utility scores for serious side effects were calculated based on these raw scores. Pravastatin, simvastatin, lovastatin, atorvastatin, rosuvastatin and fluvastatin scored 90.34, 77.56, 90.34, $61.93,94.60$, and 90.34, respectively, for this criterion.

\section{Documentations}

Available documentations could provide a good indication on the clinical experiences of patients on the drugs and counter balance the concerns that drugs which have been longer in the market will have more reported side effects. The group decided to use PubMed search 'hits' and the year of drug approved for the US market to score this criterion. ${ }^{20,29}$ The search terms used were the drug name [title], hyperlipidemias
[MeSH terms] and randomized controlled trial, reviews and meta-analysis [for publication types]. Drugs with more documentation hits and those approved for use earlier, scored higher on this criterion. Simvastatin scored 100, pravastatin and atorvastatin both scored 90, while the others scored 80 for this criterion.

\section{Drug applicability}

Number of formulations and drug strengths

The variety in formulations and strengths is considered a distinct advantage as it provides flexibility when prescribing the drugs. All the statins evaluated were available in oral tablet forms of 3 or more dosage strengths; thus, equal scores of 90 were given to all except fluvastatin. Fluvastatin was given a score of 100, as it is available in 3 strengths, of which one is a modified release (MR) formulation.

\section{Number of approved indications}

The number of approved indications provided information on the accepted evidence-based use of the drugs. Simvastatin, atorvastatin and rosuvastatin that were approved for five indications, namely for primary, mixed and familial hypercholesterolemia and for prophylaxis of cardiovascular disease (CVD) in patients with or without previous CVDs, mixed hypercholesterolemia scored full marks out of 100 for this criterion. Familial hypercholesterolemia is not a registered indication (by the Drug Control Authority of Malaysia) for pravastatin, lovastatin and fluvastatin. They were scored at 80 .

\section{Dose frequency}

Patients' compliance to prescribed drugs is of utmost importance to achieve the intended therapeutic effects. A higher daily dose frequency is often associated with reduced compliance. ${ }^{30}$ For this criterion, all the statins were given the same score of 100 based on the once daily dose commonly prescribed.

\section{Frequent, but not serious side effects}

This criterion was scored similarly to the serious side effects previously discussed using MM as the main reference. For frequent side effects four risk categories based on the MM classifications were used which are, more common (occurrences $\geq 10 \%$ ), common $(1-10 \%)$, less common $(\geq 0.1 \%$ and $\leq 1 \%$ ), and rare $(<1 \%)$. They were assigned risk factors of $2,1,0.5$ and 0.1 , respectively. Using the information on side effects for individual statins from MM, raw scores were assigned. Using Formula 2, the utility scores for this criterion were then calculated, based on these raw scores. 
Pravastatin, simvastatin, lovastatin, atorvastatin, rosuvastatin and fluvastatin scored 88.1, 72.8, 95.9, 67.7, 61.0, and 78.6, respectively, for this criterion.

\section{Interactions with food}

Advice on the dose timings with regards to meals were considered next. A score of 90 was assigned to lovastatin and simvastatin, which need to be taken after the evening meal. A full score of 100 was given to the others, without timing restrictions.

\section{Dose adjustments}

Information on dose adjustments and contraindications during pregnancy and compromised kidneys and livers were obtained from MM. All the statins reviewed were contraindicated in pregnancy and active liver disease. Dose adjustment in severe renal impairments was found to be necessary for all the statins, except atorvastatin. Accordingly, the group concurred to score 80 for atorvastatin and 70 for the other statins.

\section{Economics \\ Drug costs}

No published local pharmacoeconomic studies were found for the statins reviewed. The group decided to use drug costs per DDD to score this criterion. Drug costs used are the average wholesale costs for the Ministry of Health except for fluvastatin (not a listed drug in FUKKM) where the private wholesale cost is used. Formula 2 (scores are inversely proportional to raw data [costs]) was used and the drug costs were substituted for $f$ in the formula to obtain the utility scores for the cost criterion. The calculated scores were 93.4, 97.7, 99.9, $96.99,72.1$, and 42.5 for pravastatin, simvastatin, lovastatin, atorvastatin, rosuvastatin, and fluvastatin, respectively.

\section{Step 6: calculate the weighted utility scores}

Using the factor weights from STEP 3 and the utility score from STEP 5, the weighted scores of each criterion were calculated.

$$
\begin{aligned}
\mathrm{U}(\text { weighted })= & \text { Weight }(\text { of criteria }) \times \text { Utility Score } \\
& (\text { of criteria })=W_{c} * U_{c}
\end{aligned}
$$

When there was more than one criterion in each attribute, each attribute utility score could be obtained by adding up the weighted utility scores of all the criteria included under that attribute:

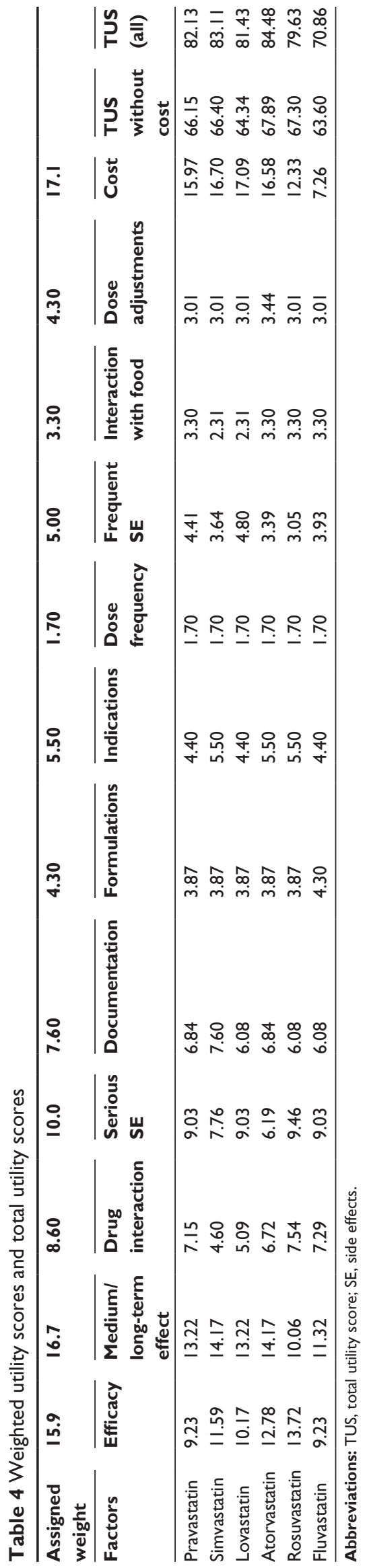




$$
U a=\sum_{c=1}^{n} W_{c} * U_{c}
$$

\section{Step 7: calculate the TUS}

Finally, the TUS for each drug was calculated by adding all the weighted utility scores (for all the criteria considered) for a particular drug.

$$
\operatorname{TUS}(\operatorname{Drug} \mathrm{A})=\sum_{\text {allcriteria }} U_{c} * W_{c}
$$

\section{Results (step 6 and step 7)}

The resultant weighted utility scores and total utility scores (TUS) of each individual statin reviewed are presented in Table 4. The TUS with cost scores and TUS without cost scores were distinguished to clearly appreciate the effects of drug costs on the drug ranking.

\section{Step 8: rank the drugs}

Drugs were ranked based on the TUS. The results have been further discussed to ensure that they are in line with current knowledge on the drug groups. Any irregularities will be clarified.

\section{Results (step 8)}

The ranking obtained for the statins reviewed in this exercise (from the highest to lowest TUS including cost scores) was atorvastatin, simvastatin, pravastatin, lovastatin, rosuvastatin and fluvastatin with TUS of 84.48, 83.11, 82.13, 81.43, 79.63 , and 70.86 , respectively. The group agreed unanimously to the ranking, based on their experience on the use of these drugs.

\section{Step 9: perform sensitivity analysis by varying assigned weights}

The working group acknowledged the sensitivity of the final scores to weights assigned to the selection criteria. Thus, the

Table 5 Sensitivity analysis: varying assigned weights

\begin{tabular}{lcll}
\hline $\begin{array}{l}\text { Assigned weights (\%) } \\
\text { Efficacy }\end{array}$ & 25 & 40 & 20 \\
Safety & 25 & 20 & 20 \\
Patient acceptability & 25 & 20 & 20 \\
Costs & 25 & 20 & 40 \\
Total utility score (ranking) & & \\
Atorvastatin & $86.5 \mathrm{I}(1)$ & $85.71(\mathrm{I})$ & $88.5 \mathrm{I}(\mathrm{I})$ \\
Simvastatin & $85.23(2)$ & $83.92(2)$ & $87.57(2)$ \\
Pravastatin & $84.50(3)$ & $81.31(4)$ & $86.28(4)$ \\
Lovastatin & $83.96(4)$ & $81.47(3)$ & $87.15(3)$ \\
Rosuvastatin & $79.94(5)$ & $78.62(5)$ & $78.27(5)$ \\
Fluvastatin & $69.67(6)$ & $68.32(6)$ & $64.19(6)$ \\
\hline
\end{tabular}

assigned weights were varied to check the robustness of the base rankings. Three different weight allocations were used for the analysis; equal weights on all four attributes, highest weight (40\%) for efficacy and highest weight (40\%) for cost. The results of the sensitivity analysis are presented in Table 5 . In all the three situations, atorvastatin was found to constantly score the highest TUS, followed by simvastatin in second place. Fluvastatin also had the lowest TUS on all occasions.

\section{Discussion}

Decisions made for formulary drug selections have great impacts on prescribing practices, patients' outcomes and ultimately health expenditures. ${ }^{31}$ However, selecting drugs for the formulary is complex. Multiple criteria of different degrees of importance need to be considered. In this study, the local application of the multiattribute decision analysis, to develop a scoring tool that can be used for drug selection in a formulary review, is demonstrated. The locally developed scoring tool is able to compare and contrast the statin drugs available in the local market based on the best evidence and consensus available through expert group discussions consisting of clinical pharmacists, a family physician, pharmacoeconomists and drug reviewers. To the best of our knowledge, this is the first time such a method is being applied for drug selection at the national level in Malaysia. This model enables all the criteria/attributes involved in evaluating the drugs to be considered and weighted accordingly, based on their importance. It also allows all the criteria to be put into perspective simultaneously and deliberated upon in an objective, systematic and transparent manner.

The four main attributes considered for drug selection are drug efficacy, drug safety, drug applicability, and cost. The group assigned the highest weight for drug efficacy followed by drug safety. This reflects the prime concerns of the committee on the value of drugs in exerting their functions over their costs and applicability.

Statins are proven efficacious in controlling hypercholesterolemia to prevent cardiovascular diseases..$^{32}$ Rosuvastatin scored the highest followed by atorvastatin and simvastatin for clinical efficacy, based on evidence of better reduction of LDL-C achieved in the population taking the drug. However, being a relatively newer drug in its therapeutic class, evidence regarding the long-term effects (clinical endpoints) of rosuvastatin are relatively less compared with the other statins resulting in lower scores for this criterion. For the overall score on efficacy, atorvastatin surpassed rosuvastatin followed closely by simvastatin.

Regarding the safety attribute, pravastatin and rosuvastatin scored higher for both drug interactions and serious side effects 
criteria. Atorvastatin, simvastatin and lovastatin are metabolized in the liver via the cytochrome P450 CYP3A4 pathway; fluvastatin primarily via CYP2C9, while pravastatin and rosuvastatin are not metabolized by these systems. ${ }^{33}$ Major metabolites of pravastatin are produced in the stomach and the small intestine rather than via the cytochrome P450-dependent metabolism in the liver. Only a small portion of pravastatin that reaches the liver is metabolized. Most is excreted unchanged in the bile. ${ }^{34}$ Rosuvastatin is not extensively metabolized and mainly excreted unchanged. ${ }^{35}$ Drugs that inhibit cytochrome $\mathrm{P} 450$ pathways like itraconazole or diltiazem can increase the plasma level of atorvastatin, simvastatin and lovastatin and increase the risk of adverse events in patients taking these combinations. This is reflected in the higher scores for pravastatin and rosuvastatin based on the drug interaction information found in MM.

The serious side effects of the statins include myopathy, rhabdomyolysis and liver toxicity. Although there were some concerns initially regarding the higher adverse events related to rosuvastatin use, the safety appraisal for statins indicated that the reporting patterns on rosuvastatin-induced adverse events were comparable to those seen with other statins and did not resemble cerivastatin which has been withdrawn from the market. ${ }^{36,37}$ The slightly higher incidences of serious side effects that were reported in MM for atorvastatin and simvastatin could be due to the greater number of occurrences of drug interactions, as mentioned. The higher number of reports could also relate to the longer duration they have been in the market. This fact, on the other hand, gives the two drugs and pravastatin a higher score on the documentation reflecting clinical experience with using them.

In terms of patient acceptability, atorvastatin and simvastatin scored slightly lower compared with the other statins for frequent side effects based on the information from MM. Mild side effects reported for the statins include gastrointestinal disturbances (flatulence, constipation, nausea, vomiting), rashes, musculoskeletal pain, headache, dizziness, insomnia, pain in the extremities, rhinitis, and depression. This is supported by other literature that has reported a poorer side-effects profile for atorvastatin. ${ }^{38}$

Based on all the weighted criteria selected by the committee, atorvastatin consistently scored highest, both before and after factoring in the drug cost. Excluding cost, rosuvastatin and simvastatin ranked second and third among the statins evaluated, mainly due to their high scores on efficacy. Rosuvastatin is available in the patented form, Crestor. The cost per DDD is 50 times more than the cheapest statin (generic lovastatin: 5 sen; there are 100 sens in one Ringgit Malaysia (RM). One RM is approximately USD 0.33 in 2012.) and eight times more than generic atorvastatin (31 sen). After factoring in the costs, rosuvastatin slid down to fifth position. However, this position can potentially change once the generic form of rosuvastatin is available. The ranking was also found to be robust as atorvastatin and simvastatin scored consistently high and fluvastatin remained with the worst score when the assigned weights were varied in the sensitivity analysis.

In this study, the main information source to evaluate the drugs was Micromedex (Thomson Healthcare Ltd), a drug information database subscribed to by the Pharmaceutical Services Divisions (PSD). It is readily available to all drug evaluators and is thus the most used as a dependable reference source by drug evaluators in PSD when reviewing and comparing drugs applied for listing into the formulary. The study demonstrated the potential of MM as a reliable preliminary data source for drug review in a practical setting when the resources and time available are constrained. In this exercise, the evaluators agreed that information provided from MM are generally sufficient, consistent and reliable to score attributes like side effects (serious and common), drug interactions and to some extent clinical end-points.

During the process of developing the tool and reviewing the drugs, the committee members welcomed the consensus nature of the decisions made within the group. They brainstormed on the drug attributes to be evaluated and finally decided upon the suitable weightages that should be assigned for each. The model allowed all the attributes considered important by the committee to be evaluated simultaneously. The committee members felt confident of the scores as they were evidence based and systematically presented. Besides improving transparency and objectivity in drug selections for the formulary, the tool also potentially improved the acceptability of decisions made in light of the bottom-up nature of the process.

\section{Limitations}

It should be noted, however, that the scoring framework prepared is merely a tool to guide drug selection deliberations and help in decision-making. It, by no means, provides a definite ranking to indicate whether a drug should or should not be listed in the formulary. The tool is also not without flaws. As the scorings are based on the evaluation of the selected drug attributes/criteria, the scores are influenced by the collection of criteria being considered. Different institutions may consider different sets of criteria that they consider important to their setting. Although efforts have been taken to ensure that all criteria come under consideration based on expert 
opinions and previous work, the criteria that have been chosen for this exercise may not be exhaustive. Additionally, the weights assigned for the criteria could also vary, based on the variety of professionals in the committee. Those concerned with purchasing and budgeting often assign greater weights to cost compared with clinical experts who may show greater preference to the clinical value of the drugs and thus give higher weightage to efficacy and safety. The weights given can thus change if other stakeholders, for example patients, pharmaceutical company representatives or academicians, are included in the committee. It should also be noted that the evaluation and scores could quickly become obsolete with new evidence and changes in drug prices. However, with the framework in place, the changes can be easily included when the need arises. It should also be noted that the scores are mainly meant to guide drug listing. When prescribing statins, the needs of the individual patient and the overall cardiovascular risks will need to be considered.

\section{Conclusion}

The multiattribute scoring tool successfully systematizes the decision variables in selecting statins for the formulary, based on evidence and group consensus. From the total utility scores calculated using the scoring tool designed, atorvastatin and simvastatin are recommended to remain in the formulary and be considered as the first-line in the treatment of hypercholesterolemia.

Using the tool, drug reviewers are able to present evidence in a more organized manner, which in turn helps the decision makers reach a more coherent and acceptable decision. The tool can easily be applied to other therapeutic groups in an effort to streamline the formulary. New evidence or changes in drug costs for example can also be easily incorporated to provide updated scores when necessary. Additionally, the tool can potentially be used when reviewing new drugs proposed for inclusions into the formulary.

\section{Acknowledgments}

We would like to thank the Director General of Health, Malaysia, for granting permission to publish this study. We are also grateful to the National Institute of Health (NIH), Ministry of Health, Malaysia, for providing financial assistance through the NIH grant (NMRR-12-77-10772). Our special thanks is due to the members of the Drug Scoring Technical Committee; Anis Talib, Aznida Abd Aziz, Azuwana Supian, Noraini Saari, Rosliza Lajis, Haarathi Chandriah, Hariana Haris, Hayati Alwani, and Lim Ming Tsuey for their time, input, and views, as well as cooperation and patience in the course of building the tool framework and gathering evidence to support the tool application.

\section{Disclosure}

The authors declare that they have no competing interests in this work.

\section{References}

1. American Society of Hospital Pharmacists. ASHP guidelines on formulary system management. Am J Hosp Pharm. 1992;49(3):648-652.

2. Pharmaceutical Services Division Ministry of Health Malaysia. Panduan Kepada Formulari Ubat KKM (Guidelines to the MOH Drug Formulary). 2nd ed. Pharmaceutical Services Division Ministry of Health Malaysia. Petaling Jaya, Malaysia. 2007:1-15.

3. Scott M, Janknegt R, Brenninkmeijer R. A prelude to the Matrix Models Supplement. Expert Opin Pharmacother. 2007;8(1):S1-S4.

4. Dyer JS, Fishburn PC, Steuer RE, et al. Multiple criteria decision making, multiattribute utility theory: the next ten years. Management Science. 1992;38(5):645-654.

5. Department for Communities and Local Government L. Multi-criteria Analysis: A Manual. London: Crown; 2009:1-168.

6. Zachry WM, Skrepnek GH. Practical pharmacoeconomics: applying multiattribute utility technology to the formulary evaluation process. Formulary. 2002;37(April):199-206.

7. Baltussen R, Niessen L. Priority setting of health interventions: the need for multi-criteria decision analysis. Cost Effectiveness and Resource Allocation. 2006;4(14):1-9.

8. Chung S, Kim S, Kim J, Sohn K. Use of multiattribute utility theory for formulary management in a health system. American J Health-Syst Pharm. 2010;67(2):128-135.

9. Janknegt R. Triptans in the treatment of migraine: drug selection by means of the SOJA method. Expert Opin Pharmacother. 2007; 8(Suppl 1):S15-S30.

10. Youngkong S, Teerawattananon Y, Tantivess S, Baltussen R. Multi-criteria decision analysis for setting priorities on HIV/AIDS interventions in Thailand. Health Research Policy and Systems/BioMed Central. 2012;10(1):2-8.

11. Goetghebeur MM, Wagner M, Khoury H, Levitt RJ, Erickson LJ, Rindress D. Evidence and Value: Impact on DEcisionMaking - the EVIDEM framework and potential applications. BMC Health Services Research. 2008;8(270):1-16.

12. Janknegt R, Scott M, Mairs J, Timoney M, McElnay J. System of Objectified Judgement Analysis (SOJA) as a tool in rational and transparent drug-decision making. Expert Opin Pharmacother. 2007; 8(Suppl 1):S5-S14.

13. Alabbadi I, Crealey G, Scott M, et al. Impact of Modified System of Objectified Judgement Analysis (SOJA) methodology on prescribing costs of ACE inhibitors. Clin. Drug Invest. 2006;26(9):485-494.

14. Chongtrakul P, Sumpradit N, Yoongthong W. ISafE and the evidencebased approach for essential medicines selection in Thailand. Essential Drugs Monitor. 2005;34:18-19.

15. Pharmaceutical Services Division and Clinical Research Centre M of HM. Malaysian Statistics on Medicines 2008. Kuala Lumpur; 2013:1-176.

16. Clauson K, Marsh W, Polen HH, Seamon MJ, Ortiz BI. Clinical decision support tools: analysis of online drug information databases. $B M C$ Medical Informatics and Decision Making. 2007;7:1-7.

17. Moore AP, Wheeldon NM, Jennings M. A formulary analysis of angiotensin II antagonists in a UK teaching hospital. Disease Management and Health Outcomes. 2002;10(6):355-362.

18. World Health Organization (WHO) [homepage on the internet]. WHO Collaborating Centre for Drug Statistics Methodology, Oslo. 2012. Available at: http://www.whocc.no/atc_ddd_index/. Accessed February 19, 2012. 
19. Grundy SM, Cleeman JI, Merz CNB, et al. Implications of recent clinical trials for the National Cholesterol Education Program Adult Treatment Panel III Guidelines. Journal of the American College of Cardiology. 2004;44(3):720-732. 3.

20. Micromedex ${ }^{\circledR}$ Healthcare Series (database on CD-ROM), Thomson Reuters, Greenwood Village, CO, USA. Volume 152 (6/2012).

21. TrialResults-centre France. Trialresultscenter.org [homepage on the internet]. Available at: http://www.trialresultscenter.org/sNb.asp. Accessed February 22, 2012.

22. Tran Y, Frial T, Miller P. Statin's cost-effectiveness: a Canadian analysis of commonly prescribed generic and brand name statins. Can J Clin Pharmacol. 2007;14(2):e205-e214.

23. Jones PH, Davidson MH, Stein E, et al. Comparison of the efficacy and safety of rosuvastatin versus atorvastatin, simvastatin, and pravastatin across doses (STELLAR Trial). The American Journal of Cardiology. 2003;93(2):152-160.

24. Kapur NK, Musunuru K. Clinical efficacy and safety of statins in managing cardiovascular risk. Vascular Health and Risk Management. 2008;4(2):341-353.

25. Edwards JE, Moore RA. Statins in hypercholesterolaemia: a dosespecific meta-analysis of lipid changes in randomised, double blind trials. BMC Fam Pract. 2003;4:18.

26. Hippisley-Cox J, Cater R, Pringle M, Coupland C. Cross sectional survey on effectiveness of lipid lowering drugs in reducing serum cholesterol concentration in patients in 17 general practices. $B M J$. 2003;326:689.

27. Ong H. Evidence-based prescribing of statins: a developing world perspective. PLoS Medicine. 2006;3(3):e50. Available at: http:// www.plosmedicine.org/article/info\%3Adoi\%2F10.1371\%2Fjournal. pmed.0030050. Accessed July 22, 2013.

28. WebMD LLD. New York. Reference.medscape.com. Available at: http:// reference.medscape.com/pharmacists. Accessed March 22, 2012.

29. US National Library of Medicines, National Institute of Health. Pubmed. gov [homepage on the internet]. Available at: http://www.ncbi.nlm.nih. gov/pubmed. Accessed March 2, 2012.

30. Claxton AJ, Cramer J, Pierce C. A systematic review of the associations between dose regimens and medication compliance. Clinical Therapeutics. 2001;23(8):1296-1310.

31. Schiff GD, Galanter WL, Duhig J, et al. A prescription for improving drug formulary decision making. PLoS Medicine. 2012;9(5):1-7.

32. Expert Panel on Detection Evaluation and Treatment of High Blood Cholesterol In Adults (Adult Treatment Panel III). Executive Summary of The Third Report of The National Cholesterol Education Program (NCEP). JAMA. 2001;285(19):2486-2497.

33. Neuvonen PJ, Niemi M, Backman JT. Drug interactions with lipidlowering drugs: mechanisms and clinical relevance. Clinical Pharmacology and Therapeutics. 2006;80(6):565-581.
34. Hatanaka T. Clinical pharmacokinetics of pravastatin: mechanisms of pharmacokinetic events. Clin Pharmakokinetics. 2000;39(6): $397-412$.

35. AstraZeneca Pharmaceuticals. Full Prescribing Information: Rosuvatatin Calcium (R). 2013:1-9.

36. Davidson MH, Clark JA, Glass LM, Kanumalla A. Statin safety: an appraisal from the adverse event reporting system. Am J Cardiol. 2006;97(8A):32C-43C.

37. Florentinus SR, Heerdink ER, Klungel OH, de Boer A. Should rosuvastatin be withdrawn from the market? Lancet. 2004;364(9445): 1577; author reply $1578-1579$.

38. Silva MA, Swanson AC, Gandhi PJ, Tataronis GR. Statin-related adverse events: a meta-analysis. Clinical Therapeutics. 2006;28(1):26-35.

39. Nakamura H, Arakawa K, Itakura H, et al. Primary prevention of cardiovascular disease with pravastatin in Japan (MEGA Study): a prospective randomised controlled trial. Lancet. 2006;368(9542): 1155-1163.

40. Shepherd J, Blauw G, Murphy M. Pravastatin in elderly individuals at risk of vascular disease (PROSPER): a randomised controlled trial. The Lancet. 2002. Available at: http://www.sciencedirect.com/science/ article/pii/S014067360211600X. Accessed July 22, 2013.

41. Heart Protection Study Collaborative Group. Articles MRC / BHF Heart Protection Study of cholesterol lowering with simvastatin in 20,536 high-risk individuals: a randomised placebo-controlled trial. Lancet. 2002;360(July 6):7-22.

42. Downs JR, Clearfield M, Weis S, et al. Primary prevention of acute coronary events with lovastatin in men and women with average cholesterol levels: results of AFCAPS/TexCAPS. [Abstract]. JAMA. 1998;279(20):1615-1622.

43. Amarenco P, Bogousslavsky J, Callahan A, et al. High-dose atorvastatin after stroke or transient ischemic attack. New Engl J Med. 2006;355(6):549-559.

44. Colhoun HM, Betteridge DJ, Durrington PN, et al. Primary prevention of cardiovascular disease with atorvastatin in type 2 diabetes in the Collaborative Atorvastatin Diabetes Study (CARDS): multicentre randomised placebo-controlled trial. Lancet. 2004;364(9435):685-696.

45. Kjekshus J, Apetrei E, Barrios V, et al. Rosuvastatin in older patients with systolic heart failure. New Engl J Med. 2007;357(22):2248-2261.

46. Ridker PM, Danielson E, Fonseca FAH, et al. Rosuvastatin to prevent vascular events in men and women with elevated C-reactive protein. New Engl J Med. 2008;359(21):2195-2207.

47. Anderssen SA, Hjelstuen AK, Hjermann I, Bjerkan K, Holme I. Fluvastatin and lifestyle modification for reduction of carotid intimamedia thickness and left ventricular mass progression in drug-treated hypertensives. Atherosclerosis. 2005;178(2):387-397. 


\section{Supplementary material}

\section{Assignments of attribute and factor weightages for multiattribute scoring tool (MAST)}

Introduction: in drug selection, degree of importance of each factor/selection criteria being considered could vary. For some individuals efficacy/effectiveness may be more important than costs, for example. Thus, there is a need to allocate weights to the factors/selection criteria based on their importance. Together with individual drug values, the assigned criteria weights will contribute to the final score calculation.

Instructions: for this exercise, we need you to weigh the drug factors/selection criteria below by allocating percentage (\%) to the factors/selection criteria listed (1a to $4 \mathrm{a}$ ). The more important the criterion is, the more \% should be allocated to it.

\section{Allocation of weightages can be done in two easy steps.}

1. In the boxes marked *, allocate $\%$ to the four attributes listed (efficacy/effectiveness, safety, drug applicability, and economics). Higher percentages should be given to attributes that you think are more important. For example if you think that efficacy/effectiveness is two times more important than economics, when economics is allocated $20 \%$, efficacy/ effectiveness should be given $40 \%$. Percentages given to the four attributes should add up to $100 \%$.

2. In the boxes marked $\#$, allocate $\%$ for the corresponding factors/selection criteria. The $\%$ allocated for the factors of each attribute should total up to the \% given to the particular attribute already allocated in Step 1.

For example if you have given $40 \%$ for efficacy/effectiveness and think that clinical efficacy is more important between the two factors, you may want to allocate $25 \%$ for clinical efficacy and $15 \%$ for effect on clinical endpoints.

\begin{tabular}{|c|c|c|c|c|c|}
\hline No & Attributes & Attribute weight (\%) & Factor no & Factors/selection criteria & Factor weight (\%) \\
\hline \multirow[t]{2}{*}{1} & Efficacy/effectiveness & $*$ & la & Clinical efficacy & \# \\
\hline & & & $\mathrm{lb}$ & Effect on clinical endpoints & \# \\
\hline \multirow[t]{3}{*}{2} & Safety & * & $2 a$ & Drug interactions & \# \\
\hline & & & $2 b$ & Rare, hazardous side effects & \# \\
\hline & & & $2 c$ & Documentation & \# \\
\hline \multirow[t]{6}{*}{3} & Drug applicability & $*$ & $3 a$ & Number of formulations available & \# \\
\hline & & & $3 b$ & Number of approved indications & \# \\
\hline & & & $3 c$ & Dosage frequency & \# \\
\hline & & & $3 d$ & Frequent side effects & \# \\
\hline & & & $3 e$ & Interaction with food & \# \\
\hline & & & $3 f$ & $\begin{array}{l}\text { Dose adjustments in special } \\
\text { population }\end{array}$ & \# \\
\hline \multirow[t]{2}{*}{4} & Economics & * & $4 a$ & Costs & \# \\
\hline & Total & $100 \%$ & & & $100 \%$ \\
\hline
\end{tabular}

Filled in by:

Email:

THANK YOU FOR YOUR TIME. IT IS HIGHLY APPRECIATED.

\section{Publish your work in this journal}

Therapeutics and Clinical Risk Management is an international, peerreviewed journal of clinical therapeutics and risk management, focusing on concise rapid reporting of clinical studies in all therapeutic areas, outcomes, safety, and programs for the effective, safe, and sustained use of medicines. This journal is indexed on PubMed Central, CAS,
EMBase, Scopus and the Elsevier Bibliographic databases. The manuscript management system is completely online and includes a very quick and fair peer-review system, which is all easy to use. Visit http://www.dovepress.com/testimonials.php to read real quotes from published authors. 